\title{
Robust Multi-Partite Multi-Level Quantum Protocols
}

\author{
Hideomi Nihira* and C. R. Stroud, Jr. \\ The Institute of Optics, University of Rochester, Rochester, NY 14627, USA
}

(Dated: August 28, 2018)

\begin{abstract}
We present a tripartite three-level state that allows a secret sharing protocol among the three parties, or a quantum key distribution protocol between any two parties. The state used in this scheme contains entanglement even after one system is traced out. We show how to utilize this residual entanglement for quantum key distribution purposes, and propose a realization of the scheme using entanglement of orbital angular momentum states of photons.
\end{abstract}

*Electronic address: nihira@optics.rochester.edu 


\section{INTRODUCTION}

Here we present a simple three-level tripartite quantum protocol that can be generalized to a N-level N-partite scheme. The initial state that the three parties share can be used for both quantum secret sharing protocol or a BB84-like protocol between any two parties. Although BB84 is a protocol in which Alice and Bob perform a measurement on the same particle, making separate measurements on two entangled particles shared between Alice and Bob can also produce perfectly correlated measurement outcomes. In this manner a BB84like scheme can be employed for entangled states. The interesting aspect of the proposed state is that, unlike the $|G H Z\rangle_{N}$ state, the reduced density matrix of any two particles still contains some entanglement, and perfectly correlated measurements can be made in the reduced space thus making the protocol robust against particle loss. We also show how this scheme can be realized using entangled orbital angular momentum states of light.

The original cryptographic protocol introduced by Bennett and Brassard [1] generated a secure key using two sets of bases that were mutually unbiased. Later, Ekert suggested the use of entangled states to generate a common key in a secure fashion 2]. However, these quantum key distribution (QKD) protocols involved only two parties and two-level systems. In recent years researchers have drawn their attention to QKD protocols that involve multi-level systems with two parties [3, 4, 5, 6, 7, 8], or multiple parties with twolevel systems 9, 10]. Motivating the pursuit of multi-level quantum key distribution is that more information can be carried by each particle thereby increasing the information flux, and some multi-level protocols have been shown to have greater security against eavesdropping attacks [3, 8]. As for multi-party protocols there is the quantum secret sharing protocol which employs $|G H Z\rangle_{N}$ states [9, 10], but there seems to be little else besides this.

On the experimental aspect, one of the obstacles for multi-level schemes is the feasibility of such schemes. Atoms have multiple energy levels that can be utilized, but preparing atoms in some prescribed state and sending them off to separate parties is not realistic. The decoherence time of the state will determine how far the particles can travel before they become useless for any scheme that requires a particular state. However, recent experimental demonstrations in the entanglement of orbital angular momentum states of photons and the generation of arbitrary entangled states with these orbital angular momentum quantum numbers [11, 12, 13] makes photons a promising resource for multidimensional quantum pro- 
tocols. Furthermore, much work has been done in detecting these orbital angular momentum states of light and its superpositions at the single photon level [14, 15, 16].

Here we investigate another possible multi-party protocol involving a state which, unlike the $|G H Z\rangle_{N}$ state, contains some entanglement even after one of the particles is traced out. Although the remaining state is a mixed state, perfectly correlated measurements can be made by making measurements in a reduced space. This makes the state rather interesting because it allows any two parties to create a key without any help from the third.

\section{QUANTUM SECRET SHARING PROTOCOL}

Suppose there is a task at hand where the involvement of more than one party is needed for the sake of checks and balances. This could be for launching missiles, opening bank safes, or other sensitive matters where no one individual can be trusted to execute. To this end, one sends only parts of the launch code, bank vault combination, etc., to each party involved in the task. The message can be deciphered only when all the parties involved cooperate. In recent years quantum mechanical version of these secret sharing protocols have been discussed using GHZ states [9, 10]. Here we propose another secret sharing scheme using a three-level system.

We assume that the three parties (Alice, Bob, and Charlie) share the state

$$
|\Psi\rangle=\frac{1}{\sqrt{6}}[(|a b\rangle+|b a\rangle)|c\rangle+(|a c\rangle+|c a\rangle)|b\rangle+(|c b\rangle+|b c\rangle)|a\rangle]
$$

where $|a\rangle,|b\rangle$, and $|c\rangle$ are the three quantum levels and $(|a b\rangle+|b a\rangle)|c\rangle$ is short hand for

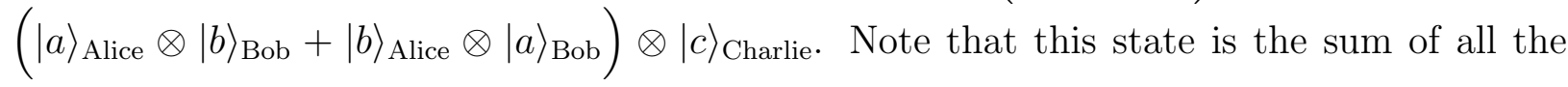
permutations of the three levels, and that the state collapses into a Bell state when one of the parties makes a measurement in the representational basis hence the measurement outcomes are perfectly correlated. Now we define another set of measurement basis vectors

$$
\begin{gathered}
|u 1\rangle=\frac{1}{\sqrt{3}}[|a\rangle+|b\rangle+|c\rangle], \\
|u 2\rangle=\frac{1}{\sqrt{3}}\left[|a\rangle+e^{i \phi}|b\rangle+e^{-i \phi}|c\rangle\right],
\end{gathered}
$$




$$
|u 3\rangle=\frac{1}{\sqrt{3}}\left[|a\rangle+e^{-i \phi}|b\rangle+e^{i \phi}|c\rangle\right]
$$

where $\phi=\frac{i 2 \pi}{3}$. This set of measurement basis vectors is a mutually unbiased basis set for a three level system. The original state is perfectly correlated in this measurement basis as well since

$$
\begin{gathered}
\langle u 1, u 1 \mid \Psi\rangle=|u 1\rangle,\langle u 2, u 1 \mid \Psi\rangle=-|u 2\rangle,\langle u 3, u 1 \mid \Psi\rangle=-|u 3\rangle, \\
\langle u 1, u 2 \mid \Psi\rangle=-e^{-i \phi}|u 3\rangle,\langle u 2, u 2 \mid \Psi\rangle=-e^{-i \phi}|u 1\rangle,\langle u 3, u 2 \mid \Psi\rangle=e^{-i \phi}|u 2\rangle, \\
\langle u 1, u 3 \mid \Psi\rangle=-e^{i \phi}|u 2\rangle,\langle u 2, u 3 \mid \Psi\rangle=e^{i \phi}|u 3\rangle,\langle u 3, u 3 \mid \Psi\rangle=-e^{-i \phi}|u 1\rangle
\end{gathered}
$$

where $\langle u 1, u 1 \mid \Psi\rangle=|u 1\rangle$ is shorthand for ${ }_{\text {Bob }}\left\langle u 1\left|\otimes_{\text {Alice }}\langle u 1 \mid \Psi\rangle=\right| u 1\right\rangle_{\text {Charlie. }}$ First, Alice measures her particle using one of the bases, then Bob makes his measurement in one of the bases and then Charlie does the same. If all the parties involved measure in the same basis, then they will keep the outcome of their measurement. At the very end, Bob and Charlie get together and compare notes to determine Alice's measurement outcomes. Clearly, from the structure of the initial state, neither Bob nor Charlie could tell what Alice's measurement was without getting together and sharing measurement results.

\section{QUANTUM KEY DISTRIBUTION PROTOCOL}

Alice, Bob, and Charlie still share the same initial state described before, but what happens if Charlie loses his particle? Can Alice and Bob still utilize the entanglement they have between their particles to communicate? There is indeed a simple way to take advantage of the residual entanglement Alice and Bob share. The reduced density matrix of the original state when Charlie's system is traced out is

$$
\hat{\rho}_{A B}=\frac{1}{3}\left[\left|\Psi_{a b}\right\rangle\left\langle\Psi_{a b}|+| \Psi_{b c}\right\rangle\left\langle\Psi_{b c}|+| \Psi_{c a}\right\rangle\left\langle\Psi_{c a}\right|\right]
$$

where $\left|\Psi_{i j}\right\rangle=\frac{1}{\sqrt{2}}[|i j\rangle+|j i\rangle]$ and $i, j \in(a, b, c)$. Alice and Bob share this mixed state, but the question remains whether they can get perfectly correlated measurement outcomes 
from this state. Indeed, this can be done if Alice and Bob restrict their measurements to a two-dimensional subspace of the three-level system.

Let us supposed Alice and Bob decide to make measurements in the $(|a\rangle,|b\rangle)$ subspace,

so they measure in either the $\{|a\rangle,|b\rangle\}$ basis or $\left\{\frac{1}{\sqrt{2}}(|a\rangle+|b\rangle), \frac{1}{\sqrt{2}}(|a\rangle-|b\rangle)\right\}$ basis. If the state they shared was $\left|\Psi_{a b}\right\rangle$, then they would get perfectly correlated measurement outcomes provided they measured in the same basis. In the case in which the state they shared was $\left|\Psi_{b c}\right\rangle$ either Alice or Bob will get a click in his or her detector if they measure in the $\{|a\rangle,|b\rangle\}$ basis since $\left|\Psi_{b c}\right\rangle$ has a component in $|b\rangle$. However, in this case it is impossible for both Alice and Bob to get a click in their detectors, since if one measures the state of the particle to be in $|a\rangle$, then the other party's particle will be in state $|c\rangle$, which is not within the two-dimensional subspace in which they are making the measurement. A similar argument holds for the $\left\{\frac{1}{\sqrt{2}}(|a\rangle+|b\rangle), \frac{1}{\sqrt{2}}(|a\rangle-|b\rangle)\right\}$ basis, it is impossible for both Alice and Bob to get a click in their detectors. Hence, for QKD purposes Alice and Bob will disregard the measurements in which: 1) they did not measure in the same basis, and 2) when they did not both register a click in their detectors. The remaining measurements they made will be perfectly correlated.

In fact, Alice and Bob don't even need to previously agree upon the subspace in which they make the measurement. They can randomly choose the subspace and add to the two previous criteria that they also disregard the measurements made in different subspaces.

\section{REALIZATION USING ORBITAL ANGULAR MOMENTUM OF LIGHT}

Although the protocol is independent of any particular realization, here we present an implementation of the protocol using orbital angular momentum states of light. We present both a method to generate the initial entangled state, and the means to detect both the orbital angular momentum states and its superposition.

It has been experimentally verified that the orbital angular momentum of a photon is conserved through spontaneous parametric down conversion, and the daughter photons are entangled in their orbital angular momentum [11]. Since there is no upper bound to the orbital angular momentum a photon can carry, it is ideal for multidimensional quantum protocols.

First, we will have to generate the state the three parties are going to share. Here we will 
use three entangled sources, a three beam coupler, three detectors, and a computer hologram to differentiate between the different orbital angular momentum states of the photon. The method used is in the same spirit as the method used to generate GHZ states from two entangled sources [17].

The entangled source of light we are going to use is generated through spontaneous parametric down conversion. Using a suitable computer generated hologram to modify the pump beam, we can produce the following orbital angular momentum entangled state [12],

$$
\left|\Psi_{\text {source }}\right\rangle=\frac{1}{\sqrt{3}}[|0,0\rangle+|1,1\rangle+|2,2\rangle] .
$$

We then take three of these sources and send one of each source's output into a three-beam coupler. At the output of the coupler we put another computer generated hologram with one dislocation and we place a single mode fiber that goes into a detector at each of the three diffraction orders as shown in Fig. 1. The hologram imparts a $\Delta l=0$ for the zeroth diffraction order, $\Delta l=1$ for the first diffraction order, $\Delta l=2$ for the second diffraction order, and so on to the input beam. The single mode fibers only couple in the lowest order orbital angular momentum states hence the detector placed in the second diffraction order will only click if the diffracted photon was originally in the $l=2$ state [11]. If all three detectors register a photon then it means that the photons that weren't detected have orbital angular momentum of $l=0, l=1$, and $l=2$, but we do not know which photon carries which state. Hence we are left with the state

$$
\begin{gathered}
\left|\Psi_{\text {tripartite }}\right\rangle=\frac{1}{\sqrt{6}}[|0,2,1\rangle+|0,1,2\rangle+|1,0,2\rangle+|1,2,0\rangle+|2,0,1\rangle+|2,1,0\rangle] \\
=\frac{1}{\sqrt{6}}[(|2,1\rangle+|1,2\rangle)|0\rangle+(|2,0\rangle+|0,2\rangle)|1\rangle+(|0,1\rangle+|1,0\rangle)|2\rangle] .
\end{gathered}
$$

This is the original state with which we started, Eq. (11), by replacing $|a\rangle,|b\rangle$, and $|c\rangle$ with $|0\rangle,|1\rangle$, and $|2\rangle$.

Now that we have the state which the three parties share, the problem we are left with is to detect the orbital angular momentum states and its superposition. This could also be done using holograms [14, 15], but it is rather inefficient and it is not particularly suitable when considering single photon states. The method of choice here is a simple interferometric scheme employing a Mach-Zehnder interferometer with Dove prisms in its path [16]. 


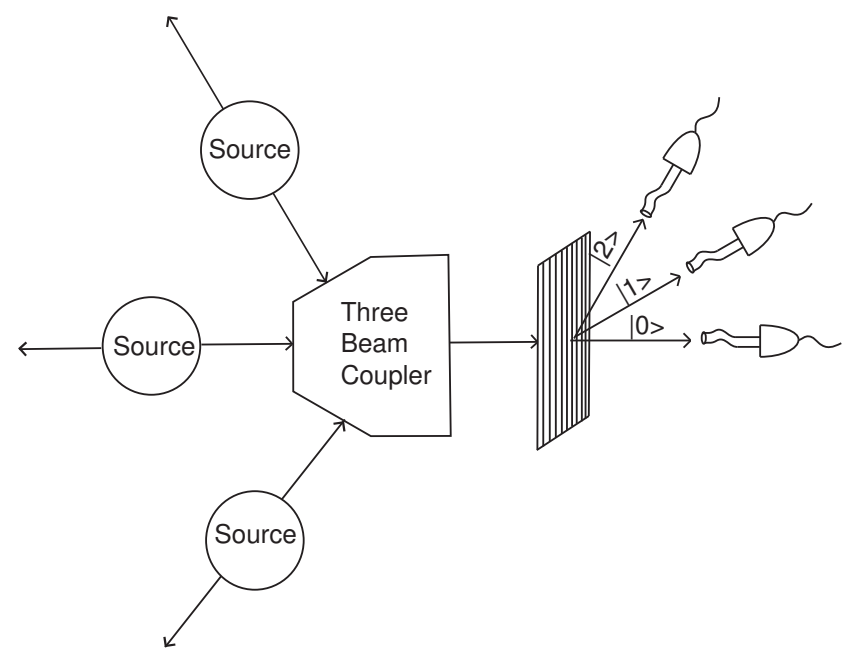

FIG. 1: Generation of tripartite three-level entangled state. The three photons that do not get detected are in the state $\left|\Psi_{\text {source }}\right\rangle$ provided all three detectors detect a photon

In the first stage the Dove prisms in the two arms are rotated with respect to one another by an angle of $\alpha / 2=\pi / 2$ (See Fig. 2). This creates a relative phase shift between the beams in the two arms of $\theta=l \pi$, where $l$ is the orbital angular momentum quantum number. The phase shift is produced because the Dove prism flips the transverse structure of the field. Since the Laguerre Gaussian modes have a $e^{i l \phi}$ phase structure, the Dove prism serves as a device that imparts a $l$-dependent phase shift. Now, by adjusting the path difference appropriately one can make it so that the odd and even orbital angular momentum states come out of the two different output ports of the interferometer. The orbital angular momentum states of the incoming beam can be sorted out by cascading these devices with different angles between the Dove prisms [16]. The photon's state can then be collapsed into a particular $l$-state by placing detectors at each of the output ports.

In detecting superposition states Eqs. (2,4), the problem comes down to determining the relative phase difference between the orbital angular momentum states. Since orthogonal states do not interfere with one another, we have to put holograms at each output port of the sorting device to convert them all into the same $l$-state. After this is done the photons are sent through a three-port interferometer where the paths are appropriately adjusted so that the three output ports are the superposition states of interest [18].

For the case when only two of the three parties want to generate a secure key the two parties use only two of the three output ports. This too is easily done with the existing 


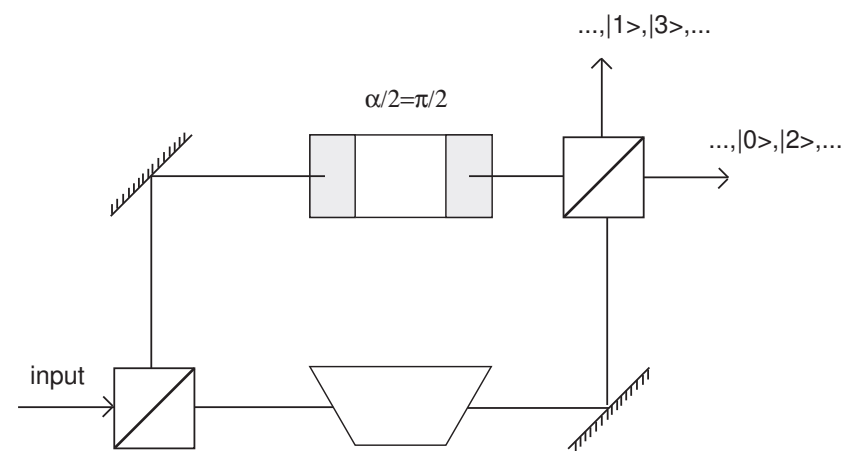

FIG. 2: Sorting orbital angular momentum states of light. The Dove prisms in the two arms are rotated with respect to one another by an angle of $\alpha / 2=\pi / 2$. With appropriate path differences the even and odd orbital angular momentum states emerge from different ports of the beam splitter.

setup. After the sorting device the two parties can measure in the orbital angular momentum basis, or its superposition in the two-dimensional space. Later, they will divulge both their measurement basis and the subspace they measured in to determine which measurements to keep.

\section{CONCLUSION}

Here we have shown a tripartite three-level system that can be used for both secret sharing protocols involving all three parties, or quantum key distribution protocol between any two parties. The two parties generate a secret key by taking advantage of the residual entanglement of the reduced density matrix. This is done by making their measurements in a reduced space. A physical realization of this scheme has also been shown through the use of entangled orbital angular momentum states of photons.

\section{Acknowledgments}

We would like to thank John Howell, Govind Agrawal, Thomas Brown, and Miguel Alonso for helpful discussions. This work was supported in part by the ARO-adminstered MURI 
Grant DAAD 19-99-1-0252.

[1] C. H. Bennett and G. Brassard, Proceedings of the IEEE International Conference on Computers, Systems and Signal Processing, Bangalore, India pp. 175-179 (1984).

[2] A. K. Ekert, Phys. Rev. Lett. 67, 661 (1991).

[3] T. Durt, N. J. Cerf, N. Gisin, and M. Żukowski, Phys. Rev. A 67, 012311 (2003).

[4] H. Bechmann-Pasquinucci and W. Tittel, Phys. Rev. A 61, 062308 (2000).

[5] M. Bourennane, A. Karlsson, G. Björk, N. Gisin, and N. J. Cerf, J. Phys. A 35, 10065 (2002).

[6] M. Bourennane, A. Karlsson, and G. Björk, Phys. Rev. A 64, 012306 (2001).

[7] N. J. Cerf, M. Bourennane, A. Karlsson, and N. Gisin, Phys. Rev. Lett. 88, 127902 (2002).

[8] D. Bruß, Phys. Rev. Lett. 81, 3018 (1998).

[9] M. Hillery, V. Buzek, and A. Berthiaume, Phys. Rev. A 59, 1829 (1999).

[10] V. Scarani and N. Gisin, Phys. Rev. A 65, 012311 (2001).

[11] A. Mair, A. Vaziri, G. Weihs, and A. Zeilinger, Nature 412, 313 (2001).

[12] J. P. Torres, Y. Deyanova, L. Torner, and G. Molina-Terriza, Phys. Rev. A 67, 052313 (2003).

[13] S. Franke-Arnold, S. M. Barnett, M. J. Padgett, and L. Allen, Phys. Rev. A 65, 033823 (2002).

[14] A. Vaziri, G. Weihs, and A. Zeilinger, J. Opt. B 4, 47 (2002).

[15] V. V. Kotlyar, V. A. Soifer, and S. N. Khonina, J. Mod. Opt. 44, 1409 (1997).

[16] J. Leach, M. J. Padgett, S. M. Barnett, S. Franke-Arnold, and J. Courtial, Phys. Rev. Lett. 88, 257901 (2002).

[17] A. Zeilinger, M. A. Horne, H. Weinfurter, and M. Żukowski, Phys. Rev. Lett. 78, 3031 (1997).

[18] M. Żukowski, A. Zeilinger, and M. A. Horne, Phys. Rev. A 55, 2564 (1997). 